\title{
Metrics for Uncertainty Analysis and Visualization of Diffusion Tensor Images
}

\author{
Fangxiang Jiao ${ }^{\star}$, Jeff M. Phillips ${ }^{\star \star}$, Jeroen Stinstra ${ }^{\star \star \star}$, Jens Krger $^{\dagger}$, Raj Varma ${ }^{\ddagger}$, \\ Edward Hsu ${ }^{\S}$, Julie Korenberg ${ }^{\mathbb{I I}}$, and Chris R. Johnson $\|$
}

\begin{abstract}
In this paper, we propose three metrics to quantify the differences between the results of diffusion tensor magnetic resonance imaging (DT-MRI) fiber tracking algorithms: the area between corresponding fibers of each bundle, the Earth Mover's Distance (EMD) between two fiber bundle volumes, and the current distance between two fiber bundle volumes. We also discuss an interactive fiber track comparison visualization toolkit we have developed based on the three proposed fiber difference metrics and have tested on six widely-used fiber tracking algorithms. To show the effectiveness and robustness of our metrics and visualization toolkit, we present results on both synthetic data and high resolution monkey brain DT-MRI data. Our toolkit can be used for testing the noise effects on fiber tracking analysis and visualization and to quantify the difference between any pair of DT-MRI techniques, compare single subjects within an image atlas.
\end{abstract}

\section{Introduction}

After the invention of Diffusion Tensor magnetic resonance imaging (DT-MRI) [1], a number of fiber tractography algorithms [2-7] have been proposed over the last decade. The issues of noise, motion effects or imaging artifacts create a certainty degree of uncertainty for fiber algorithms and may produce misleading tracking results. However, quantifying and effectively visualizing the accuracy and the uncertainty between results of different fiber tracking algorithms remains a significant challenge. For quantification, many fiber bundle difference metrics have been proposed [8,9], most of which use a Euclidean distance measure based upon predefined correspondences. One problem with the distance metrics is that it is easily disturbed by the predefined correspondences, with being overestimated or underestimated, as shown in Section 3. In addition, most difference metrics do not take into account the local fiber directional information and the local fiber probability information, i.e. the fraction of fibers that pass through that voxel. This will overweight the peripheral or tail voxels and ignore the directional information

^ The Scientific Computing and Imaging Institute, University of Utah. Email: fjiao@ sci.utah.edu

$\star \star$ The School of Computing, University of Utah. Email: jeffp@ cs.utah.edu

$\star \star \star$ Numira Biosciences. Email: jeroen@ sci.utah.edu

$\dagger$ DFKI, MMCI, Saarbrcken. Email: jens.krueger@dfki.de

* The School of Computing, University of Utah. Email: varmanitw@ gmail.com

$\S$ The Department of Biomedical Engineering, the University of Utah. Email: edward.hsu@utah.edu

II The Brain Institute, Department of Pediatrics, University of Utah. Email: Julie.Korenberg@hsc.utah.edu

\| The Scientific Computing and Imaging Institute, University of Utah. Email: crj@sci.utah.edu 
of the local diffusion profile. Recently, Wassermann et al. [10] put forward a Bayesian framework based on Gaussian Processes, which takes into account prior information about the fiber structure. Unfortunately, this method assumes the distribution of the fiber point position is Gaussian, which may not always to be true. In this paper we proposed three similarity metrics: the area between corresponding fiber bundles, the Earth Mover's Distance between two fiber bundle volumes, and the current distance between two fiber bundle volume that can help better quantify differences between fiber bundles and better understand uncertainty associated with fiber tracking algorithms.

Visualization of error and uncertainty is a growing area with important applications in science, engineering and medicine [11]. However, there are very few works addressing the visualization of uncertainty or the accuracy of tensor fields and specifically of fiber tracking algorithms. A recent paper by Brecheisen et al. [12], studies how to effectively visualize how the stopping criteria of FACT algorithm(Fiber Assignment by Continuous Tracking), can influence the fiber tracking results. However, this study primarily illustrates the quantification of the difference using a single algorithm and does not provide methods for inter-algorithm comparisons. Furthermore, Brecheisen et. al. use a technique in which seed points were placed manually by expert users. Such manual placement can influence the outcome of the fiber tracking algorithm and is somewhat time consuming. In this paper we describe an interactive uncertainty visualization toolkit. Users can choose different fiber tracking algorithms, change the tracking criteria, change how seed points are distributed. Furthermore, our toolkit provides the ability to track uncertainties within different anatomical regions, easily observe areas of high uncertainty and interactively explore such high uncertainty regions locally.

\section{MATERIALS AND METHODS}

\subsection{Data}

Synthetic data: The synthetic data used in this paper was simulated by Numerical Fiber Generator (NFG) [13]. One B0 image $\left(b=0 s \cdot \mathrm{mm}^{2}\right)$ and twenty diffusion weighted images $\left(b=3000\right.$ s. $\left.\mathrm{mm}^{2}\right)$ were obtained. The image resolution is $0.1 \mathrm{~mm} \times 0.1 \mathrm{~mm} \times$ $0.1 \mathrm{~mm}$ and the image matrix size is $20 \times 20 \times 20$ voxels.

High resolution monkey brain data: The monkey brain used in this study is the right hemisphere of a whole brain. Imaging experiments were conducted on a Bruker Biospec 7-T horizontal-bore system (Bruker Inc, Billerica, MA). For data acquisition, a standard 3D diffusion-weighted spin-echo sequence was used (TR $375 \mathrm{~ms}$, TE 26 $\mathrm{ms}$, field of view $70 \times 51 \times 51 \mathrm{~mm}$, Matrix $233 \times 170 \times 170$ which yielded an isotropic resolution of 300 microns, b-value is $2,000 \mathrm{~s} / \mathrm{mm}^{2}$ ).

Adding noise: To test the robustness of our toolkit, different levels of artificial Rician noise were added to the synthetic and the monkey brain diffusion weighted images. Six signal-to-noise (SNR) ratio levels of noise are 96,48,32,24,19 and 16, which corresponds to about $2 \%, 4 \%, 6 \%, 8 \%, 10 \%$ and $12 \%$ measured by the noise mean and divided by the signal mean. To guarantee the distribution of added noise is Rician, we proceed as follows: take the Fourier transform of the diffusion weighted image, add Gaussian noise in both the real and imaginary part of, take the magnitude of the Gaussian noise disturbed complex image, and implement the inverse Fourier transform of the magnitude image to obtain the noisy image. The same procedure was used for both synthetic data and monkey brain data. One issue that needs to be specified is that the 
smoothed monkey brain data was treated as the ground truth, and different levels of noise were added directly to it. This is because there is no ground truth available for real brain data and the main focus of this paper is on how to quantify and visualize the uncertainties rather than the noise issue itself.

\subsection{Fiber Tracking Algorithms and Tracking Parameters}

In this study, we implement six algorithms, five deterministic ones: the Streamline, Tensorline, Tensor Deflection (Tend), Guided and Fast Marching algorithm, and one probabilistic algorithm: Stochastic Tractography.

The Streamline algorithm starts from seed points and integrates along the the major eigenvector direction to form the fiber tracts. The Tensorline algorithm integrates along the following outgoing vector direction: $v_{\text {out }}=f e_{1}+(1-f)\left((1-g) v_{\text {in }}+g D \cdot v_{\text {in }}\right)$, which is the weighted sum of the major eigenvector direction of the current voxel $e_{1}$ and the previous voxel $v_{\text {in }}$, and the deflection term $D \cdot v_{\text {in }}$. Weinstein et al. [3] used a linear anisotropy measure as $f$, and named the technique the Tensorline algorithm. Lazar et al. [4] extended this idea to set $f$ and $g$ to any user defined number between 0 and 1 , this is the Tend algorithm. It is worth noting that when $f=1$, both the Tensorline algorithm and the Tensor Deflection algorithms are exactly the same as the Streamline algorithm. The Guided tracking algorithm integrates along the major eigenvector direction while being guided by a priori information, which can be anatomical knowledge or fiber tracking results from some other algorithms. The Fast Marching algorithm is based on a fast marching level set method where a front interface propagates in directions normal to itself with a non-negative speed function. From this speed function, threedimensional time of arrival maps generated, which produce the connection paths among brain regions. The Stochastic fiber tracking algorithm calculates the probabilities of connections based on a Bayesian framework. To facilitate the comparisons, we use the same start and end region for all of the six algorithms. We use linear anisotropy (CL) rather than fractional anisotropy (FA) as the anisotropy value for tracking. The reason for this choice is that the tensor shape with high FA, i.e disks, do not necessarily have a clear contrast between the major and secondary eigenvalue, in which case major eigenvector direction may easily change by 90 degrees based primarily on noise effects. The step size was chosen to be $0.05 \mathrm{~mm}$ for the synthetic data, and $0.15 \mathrm{~mm}$ for the monkey brain data, while the stopping criteria was $C L=0.1$ for both synthetic data and monkey brain data. For all of the six algorithms, only fiber tracts starting from the seed region and ending in the end region are selected for comparison.

\section{Fiber Similarity Metrics}

In this section we define three distance measures between pairs of fibers $A$ and $B$, as well as between fiber bundles $\mathcal{A}=\left\{A_{1}, A_{2}, \ldots\right\}$ and $\mathcal{B}=\left\{B_{1}, B_{2}, \ldots\right\}$. Each fiber is described by a sequence of points, that is fiber $A=\left\langle a_{1}, a_{2}, \ldots\right\rangle$. We can also represent a fiber $A$ by a piecewise-linear curve defined by segments $a_{i} a_{i+1}$ between consecutive fiber points. More conveniently, we can just denote a set of voxels that a fiber goes through. For a fiber $A$, denote this set of voxels as $\bar{A}=\left\{\bar{a}_{1}, \bar{a}_{2}, \ldots\right\}$ and for a fiber bundle $\mathcal{A}$ it is denoted $\overline{\mathcal{A}}=\left\{\bar{a}_{1}, \bar{a}_{2}, \ldots\right\}$. Given a fiber bundle $\mathcal{A}$, for each voxel $\bar{a}_{h}$, we can then determine the fraction of fibers that pass through that voxel (the probability), denoted 
as $P_{\bar{a}_{h}}$. Additionally, we can calculate the average tangent direction of the fibers that pass through a voxel $\bar{a}_{h}$, denoted as $T_{\bar{a}_{h}}$. These quantities will be useful in the distance measures we define for comparing fibers and fiber bundles.

Before we introduce the new measures, we first comment on commonly used distance measures in the literature. Given two fibers $A$ and $B$, let the pointwise-order distance of the common area be defined $D$ po $(A, B)=\sum_{i=1}\left\|a_{i}-b_{i}\right\|$. Let $B_{\ell}$ denote the point on the piecewise-linear curve of fiber $B$ a distance $\ell$ from the start by arclength, and let $\ell_{A}(a)$ be the distance from the start of fiber $A$ to a point $a \in A$. Then let the corresponding arc-length distance be defined $D_{\text {cal }}(A, B)=\sum_{i=1}\left\|a_{i}-B_{\ell_{A}\left(a_{i}\right)}\right\|+\sum_{j=1} \| b_{j}-$ $A_{\ell_{B}\left(b_{j}\right)} \|$. Let $\phi_{B}(a)$ be the closest fiber point in $B$ to point $a$. Then let the corresponding closest point distance be defined $D_{\operatorname{ccp}}(A, B)=\sum_{i=1}\left\|a_{i}-\phi_{B}\left(a_{i}\right)\right\|+\sum_{j=1} \| b_{j}-$ $\phi_{A}\left(b_{j}\right) \|$. These measures are illustrated in Figure 1 of two fibers $A$ and $B$. Although, these distances may be easy to compute, they typically take the sum or the average of distances between points, which are overestimates or underestimates of the true distances. This is due either to poor predefined correspondences, poor discretization or a complex local configuration of the fibers or fiber bundles.

For the crossing point of Fiber A and Fiber B in Figure 1, the local difference value assigned to this point for any Euclidean distance measure will be zero. Although the spatial locations of the crossing point are the same, the fiber directions at this point are different for Fiber A and Fiber B. As such, the local difference value at this point should not be zero. The area difference metric defined in Section 3.1 solves this dilemma. This local area difference metric can help to visualize the local fiber difference in a more robust way based on the spatial information. For the Earth Movers Distance and the current distance, the predefined correspondences are not needed. Therefore the problem of poor predefined correspondences, poor discretization or a complex local configuration of the fibers or fiber bundles can be successfully avoided. Furthermore, when the local fiber probability or the local fiber directional information are taken into account, this will further reduce the bias by only considering the spatial location. Thus, these two global metrics are more applicable for purpose of quantifying distances accurately.

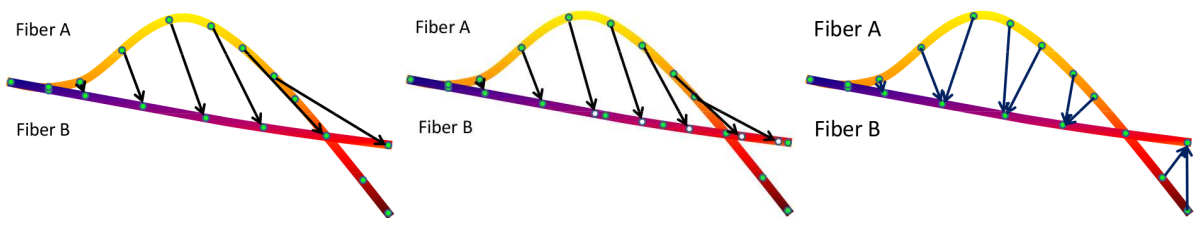

Fig. 1. Different distances: (left) $D_{\mathrm{po}}(A, B)$, (middle) $D_{\mathrm{cal}}(A, B)$, (right) $D_{\mathrm{ccp}}(A, B)$.

\subsection{The Area Between Corresponding Fibers or Corresponding Points}

We propose a distance measure $D_{\text {Area }}(A, B)$ that measures the distance between two fibers $A$ and $B$ by the area between them. Let $\operatorname{Area}(a, b, c)$ describe the area of the triangle between points $a, b$, and $c$. Let $\psi_{B}\left(a_{i}\right)$ and $\psi_{A}\left(b_{j}\right)$ describe the mappings to points in fiber $B$ and $A$, respectively, defined by the discrete Frèchet correspondence [14]; the 
closest distance from each point to the other fiber that also preserves the ordering along the fibers. Formally

$$
D_{\text {Area }}(A, B)=\sum_{i=1} \sum_{b_{j}, b_{j+1} \in \psi_{B}\left(a_{i}\right)} \operatorname{Area}\left(a_{i}, b_{j}, b_{j+1}\right)+\sum_{j=1} \sum_{a_{i}, a_{i+1} \in \psi_{A}\left(b_{j}\right)} \operatorname{Area}\left(b_{j}, a_{i}, a_{i+1}\right) .
$$

We can also assign a local distance measure at each point $a_{i} \in A$ as

$$
\begin{aligned}
D_{\text {Area }}\left(a_{i}, B\right)= & \frac{1}{2} \cdot\left[\frac{1}{2} \operatorname{Area}\left(a_{i-1}, a_{i}, \psi_{B}^{-}\left(a_{i}\right)\right)+\sum_{b_{j}, b_{j+1} \in \psi_{B}\left(a_{i}\right)} \operatorname{Area}\left(a_{i}, b_{j}, b_{j+1}\right)\right. \\
& \left.+\frac{1}{2} \operatorname{Area}\left(a_{i}, a_{i+1}, \psi_{B}^{+}\left(a_{i}\right)\right)\right],
\end{aligned}
$$

where $\psi_{B}^{-}\left(a_{i}\right)$ (resp. $\psi_{B}^{+}\left(a_{i}\right)$ ) is the min (resp. max) index point in $\psi_{B}\left(a_{i}\right)$. We use multiple terms for each point and divide by two so the local distance is symmetric (from $A$ to $B$ or $B$ to $A$ ) and the sum or the average of local distances is the global distance.

\subsection{The Earth Mover's Distance}

The Earth Mover's Distance, also called Kantorovich-Wasserstein distance, can be visualized as finding the optimal way to move piles of "earth" or dirt to fill a series of holes, minimizing the total "work" or mass times distance [15]. Based on the voxelsize representation $\overline{\mathcal{A}}$ and $\overline{\mathcal{B}}$ of fiber bundles $\mathcal{A}$ and $\mathcal{B}$, the Earth Mover's Distance between two fiber bundles is defined as

$$
\operatorname{EMD}(\overline{\mathcal{A}}, \overline{\mathcal{B}})=\frac{\sum_{i \in \overline{\mathcal{A}}} \sum_{j \in \overline{\mathcal{B}}} c_{i j} f_{i j}}{\sum_{i \in \overline{\mathcal{A}}} \sum_{j \in \overline{\mathcal{B}}} f_{i j}}=\frac{\sum_{i \in \overline{\mathcal{A}}} \sum_{j \in \overline{\mathcal{B}}} c_{i j} f_{i j}}{\sum_{j \in \overline{\mathcal{B}}} \bar{b}_{j}},
$$

where $c_{i j}$ is the cost to move a unit of supply from $i \in \overline{\mathcal{A}}$ to $j \in \overline{\mathcal{B}}$, and $f_{i j}$ is the flow that minimizes the overall cost

$$
\sum_{i \in \overline{\mathcal{A}}} \sum_{j \in \overline{\mathcal{B}}} c_{i j} f_{i j}
$$

subject the following constraints:

$$
f_{i j} \geq 0 i \in \overline{\mathcal{A}}, j \in \overline{\mathcal{B}} ; \quad \sum_{i \in \overline{\mathcal{A}}} f_{i j}=\overline{b_{j}} j \in \overline{\mathcal{B}} ; \quad \sum_{j \in \overline{\mathcal{B}}} f_{i j} \leq \bar{a}_{i} i \in \overline{\mathcal{A}},
$$

where $\bar{a}_{i}$ is the total supply of supplier $i$ and $\overline{b_{j}}$ is the total capacity of consumer $j$. In this case, they both are the probability values at the $i$ th voxel of fiber bundle $\overline{\mathcal{A}}$ and $j$ th voxel of fiber bundle $\overline{\mathcal{B}}$. The cost function $c_{i j}$, which can be any predefined distance measure in any dimension, is the Euclidean distance between the fiber voxels of two fiber bundles in this paper. Therefore, the Earth Mover's Distance between two fiber bundles is the minimum effort to redistribute the probability of one fiber bundle to match the other. This measure not only takes into account the Euclidean distance but also considers the fiber probability difference as well. 


\subsection{The Current Distance}

The current distance was proposed by Glanués and Vaillant [16] as a measure to compare a broad class of shapes (including point sets, curves, and surfaces) by how they interact with each other. Recently, Durrleman et. al. [17] investigated medical application in more depth and showed that the current distance is increasing with decreasing signal-to-noise ratio of the image. The measure can be interpreted as implicitly lifting each shape to a single point in a high (often infinite) dimensional Euclidean space, specifically, a reproducing kernel Hilbert space, where the similarity can be measured as the Euclidean distance. As such, fiber bundles can be interpreted as a set of curves, and the high dimensional vectors corresponding to each curve can be summed to create a single point representing a fiber bundle. This provides a natural distance to compare fiber bundles. Furthermore, Joshi et al. [18] showed that we can approximate the current distance between shapes arbitrarily well by a fine enough discretization. Thus, for computational reasons, we approximate each fiber $A$ by the set of voxels $\bar{A}$ it passes through. Then the similarity between two fibers can be written as

$$
\kappa(A, B)=\sum_{i} \sum_{j} K\left(a_{i}, b_{j}\right)\left(T_{\bar{a}_{i}} \cdot T_{\bar{b}_{j}}\right),
$$

where $K(a, b)$ is a kernel function (we use the Gaussian kernel with the bandwidth $h$ the same as the voxel size) and $\left(T_{\bar{a}_{i}} \cdot T_{\bar{b}_{j}}\right)$ is the dot product between two tangent vectors. Now the current distance is defined as

$$
C D(A, B)=\kappa(A, A)+\kappa(B, B)-2 \kappa(A, B) .
$$

When using a fiber bundle $\mathcal{A}=\left\{A_{1}, A_{2}, \ldots, A_{n}\right\}$ instead of a single fiber $A_{i}$, we can compute the similarity between two fiber bundles as

$$
\kappa(\mathcal{A}, \mathcal{B})=\sum_{A_{l} \in \mathcal{A}} \sum_{a_{i} \in A_{l}} \sum_{B_{h} \in \mathcal{B}} \sum_{b_{j} \in B_{h}} K\left(a_{i}, b_{j}\right)\left(T_{\bar{a}_{i}} \cdot T_{\bar{b}_{j}}\right) .
$$

Because the similarity function $\kappa$ is a summation over terms, we can accumulate the total number of fibers that pass through each voxel and take their average tangent vector in each voxel, and then we can treat each (now weighted) voxel as a single point of the fiber bundle. The self-similarity of a fiber $\kappa(A, A)$ or of a fiber bundle $\kappa(\mathcal{A}, \mathcal{A})$ can be viewed as a norm of that fiber or fiber bundle, denoting how large that shape is in the high-dimensional vector space. Alternatively, the current distance between two fibers (or fiber bundles) can be seen as the difference in how the fibers act on the underlying space, measured by how they act on each other. This action is described by its local influence in the space by the kernel function $K$ and in the direction it flows through the tangent vector. Thus the current distance measures the difference in how two fibers (or fiber bundles) flow through a given space.

\section{RESULTS AND DISCUSSION}

\subsection{Fiber Track Difference Quantification}

Figure 2 shows the tracking results of the Streamline, Fast Marching, Guided and the Stochastic tracking algorithm on synthetic data and on the monkey brain data. Since the 
Tensorline and the Tend method yield similar results to the Streamline algorithm, we only show the Streamline algorithm result. The Stochastic tracking result is embedded in each of the other three results as a semi-transparency isosurface. The colormap shows the local fractional anisotropy (FA) value. The start seed points are shown by the smaller spheres while the ending region points are shown by the larger spheres. Figure 3 shows

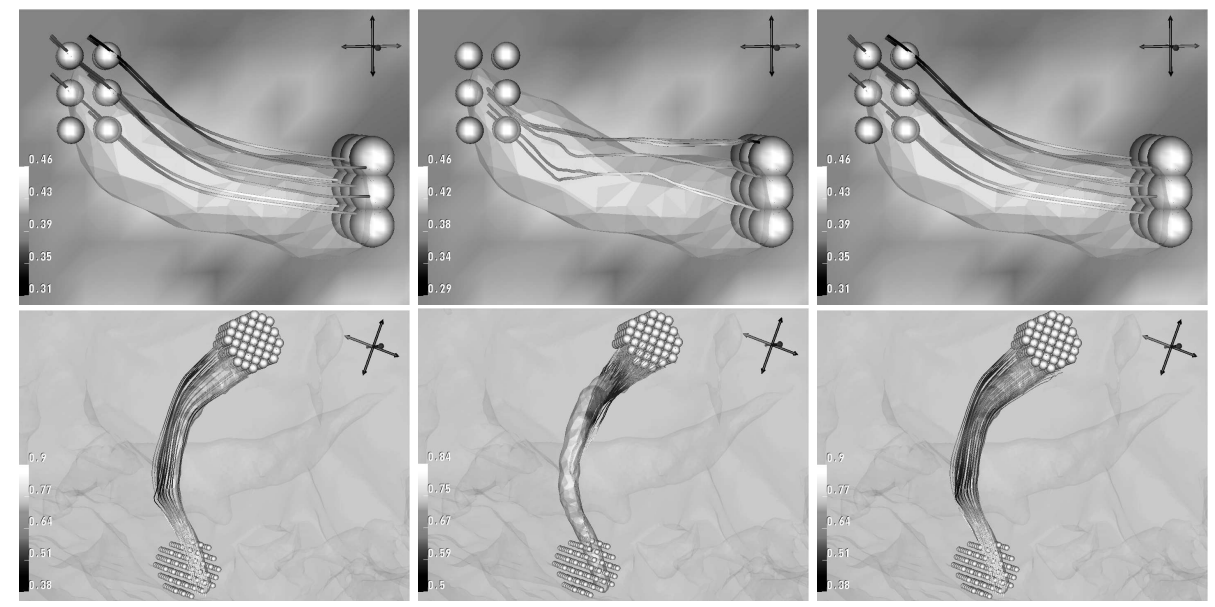

Fig. 2. The results for synthetic data (top) and monkey brain (bottom) of four tracking algorithm, Streamline (left), Fast Marching, (middle), Guided tracking (right), Stochastic tracking (embedded as isosurface), the larger sphere shows the end points, and the smaller spheres show the starting points.

the average closest distance ( $D_{\mathrm{ccp}}$ ) and average area between corresponding fibers of noise free volume and each level of noisy volume using four algorithm: Streamline, Tensorline, Guided and Tend algorithm, whose correspondence between fibers or points are easily defined. For the synthetic data, the tracking results from each algorithms are compared with the ground truth, and for the monkey brain data, the tracking results of each algorithms under different noise levels are compared with its own tracking result on the smoothed data without artificial Rician noise. One can see that either the average distance or the average area difference increases with the increasing noise level. The performance of these four algorithms are very similar, except the Guided tracking algorithm yields slightly different results from the other three methods. The fiber difference quantification using the current distance and the Earth Mover's Distance for both synthetic and monkey brain data are shown in Figure 4. The fiber tracks generated using all of the six tracking algorithms are compared with the ground truth or smoothed monkey brain data. We can see that the Stochastic tracking algorithm is very stable at different noise levels and produces the smallest difference for both measures on both data sets, while the performance of Fast Marching Method is not stable and tends to produce quite different results from the the ground truth or smoothed monkey brain data. These comparisons suggest that the Stochastic tracking algorithm is less sensitive to noise, since the noise effects are already accounted for during fiber tracking process. Furthermore, 
this suggests that the Stochastic fiber tracking algorithm may be good at finding the major structure of the data set, even at a very low signal to noise ratio. The Earth Mover's Distance and current distance can effectively capture the level of uncertainty for most of the algorithms, and the distances tend to increase when the noise level increase.
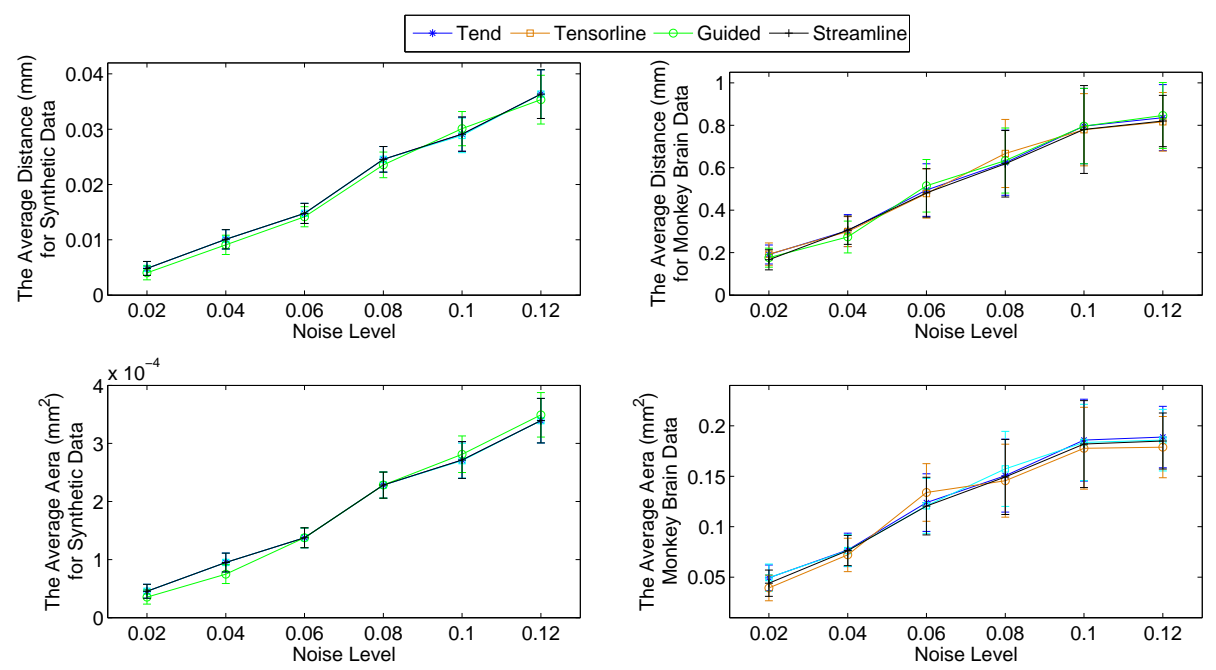

Fig. 3. The average distance (top) and average area (bottom) between fiber tracking results of the noise free volume and each level of the noisy volume for synthetic data (left) and monkey brain data (right).

Although further detailed validation is required, the three metrics put forward in this study show the potential for quantifying the difference between fibers. The area difference is good at local uncertainty visualization and quantification, which we will address in the next subsection, however it needs predefined correspondence. Both the Earth Mover's Distance and the current distance are global measures, but do not need any correspondences. Therefore, the combination of these metrics can help to quantify the uncertainty or accuracy both locally and globally.

\subsection{DT-MRI Uncertainty Visualization Toolkit}

The interactive uncertainty visualization toolkit we designed to visualize the differences between different fiber tracking algorithms, noise levels, and fiber difference metrics was created using the SCIRun problem solving environment (http://www.sci.utah.edu/ software.html). After choosing two DT-MRI volumes to be compared, a user can select fiber tracking algorithms, tracking parameters such as the stopping criteria, the interpolation method and the integration method, etc. The available tracking algorithms are the six algorithms discussed previously. We note that due to computational costs, the Fast Marching and Stochastic algorithms cannot be currently used in interactive mode. The interpolation methods in the toolkit are nearest neighbor, linear, B-spline, CatmullRom, and Gaussian interpolation. An Euler method, as well as forth-order Runge-Kutta 

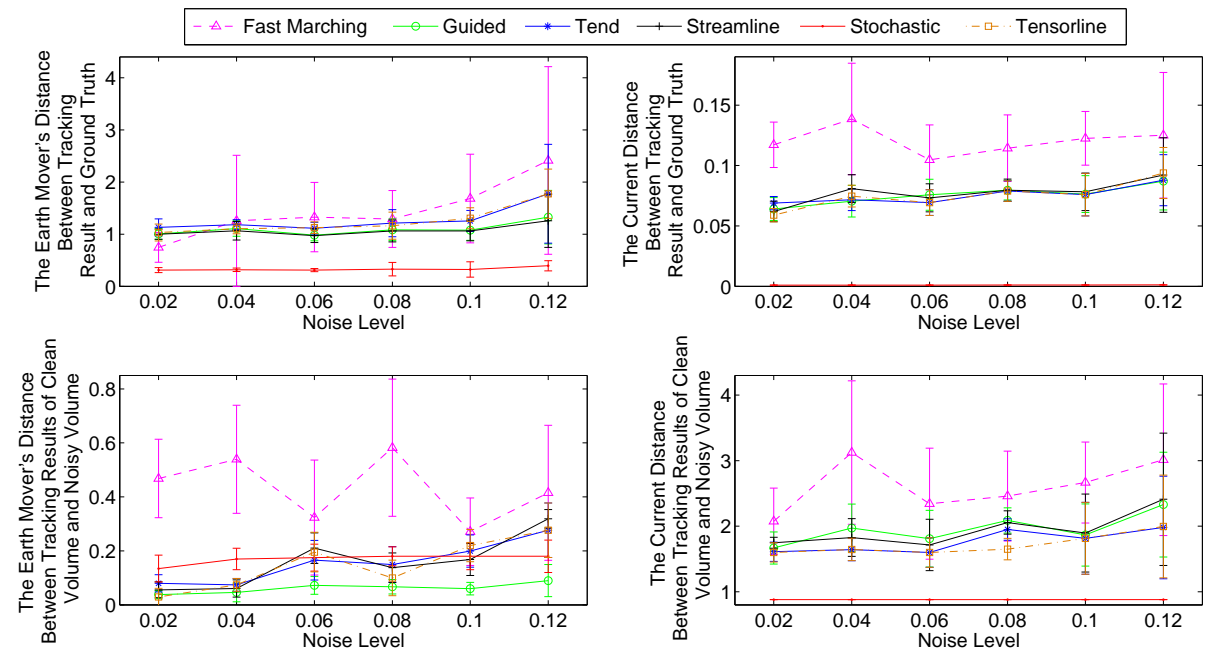

Fig. 4. The fiber difference quantification using Earth Mover's Distance (left) and current distance (right) on synthetic data(top) and monkey brain data (bottom).

integration methods are used to generate the fiber tracks. The stopping criteria includes, the threshold for the length of the fiber, the local anisotropy value, the local curvature, and the number of integration steps. The user can move a widget inside the DT-MRI volume, the position of the seed points will be linearly interpolated along the widget, and the local area difference between two preselected volumes will be interactively visualized. Furthermore, the length of the widget, the shape of the widget and the seed points density can also be changed interactively. Then correspondence of fibers between any two volume is defined by whether the fibers come from the same seed points. Figure 5 illustrates the global and local visualization windows. The left hand side shows the interactive uncertainty visualization of the synthetic data, the middle column shows the interactive uncertainty visualization of the monkey brain data and the right column shows the zoom in view of the monkey brain data. The fiber tracks are generated using the Streamline algorithm. The global and local difference histograms are shown through an attached UI interface, and the local difference histogram (in red) is updated interactively. Through this interactive UI, the user can easily compare the uncertainty or accuracy of the current fiber track with fiber tracks from different anatomical regions, which helps quickly locate areas with high uncertainty.

In general, the end points of the fibers have a larger uncertainty due to the accumulated tracking error. As shown in Figure 5, these areas are highlighted and easily located by the average area metric rather than average closesest distance metric, especially within the monkey brain data. One can also notice that the area with high uncertainty is located to the right and towards the end of the tracking for the monkey brain. While this area is visible in the distance difference visualization, it is more clearly highlighted through the local area difference visualization upon closer inspection at the right column. Taken together, a user can interactively explore, quantify, and visualize uncertainties within DTI-MR data using the our uncertainty visualization toolkit. We note 
that noise is only one of many potential DTMRI uncertainty sources. Imaging artifacts, partial voluming or even different ber tracking parameters can also produce uncertainties. Although we only focus on the uncertainty associated with different levels of noise, the toolbox we developed in this study can be used as a tool to quantify and visualize any kind of uncertainty.

\section{CONCLUSION AND FUTURE WORK}

In this paper, we put forward three metrics to quantify the difference between two fiber bundles. The quantification results on synthetic data and the monkey brain data show that the area between corresponding fibers can effectively capture the local or global uncertainty. The Earth Mover's Distance, which considers the local fiber probability, also shows good quantification of the fiber difference. The current distance metric, which considers the local fiber probability, the local fiber directional information illustrates the power of quantifying the global uncertainty. Based on all of these metrics, we illustrated an interactive uncertainty visualization toolkit within the SCIRun environment that includes six fiber tracking algorithms were implemented and associated tracking parameter and noise level options. The location and the density of the seed points can be changed interactively, and most importantly, the uncertainties can be visualized interactively and quantitatively compared with the fiber tracks in different anatomical regions. Thus our toolkit facilitates DT-MRI tracking algorithm comparison, the impact of noise or other artifacts, and visual uncertainty localization.

Currently, we are working on the analysis of the fiber differences between subjects from different age groups within a human brain atlas, which will quantify the variabilities of the fiber tracking results for different age groups. In future, we will apply the metrics defined in this study to fiber clustering and segmentation, which may potentially improve fiber clustering and segmentation accuracy. Fiber bundle difference

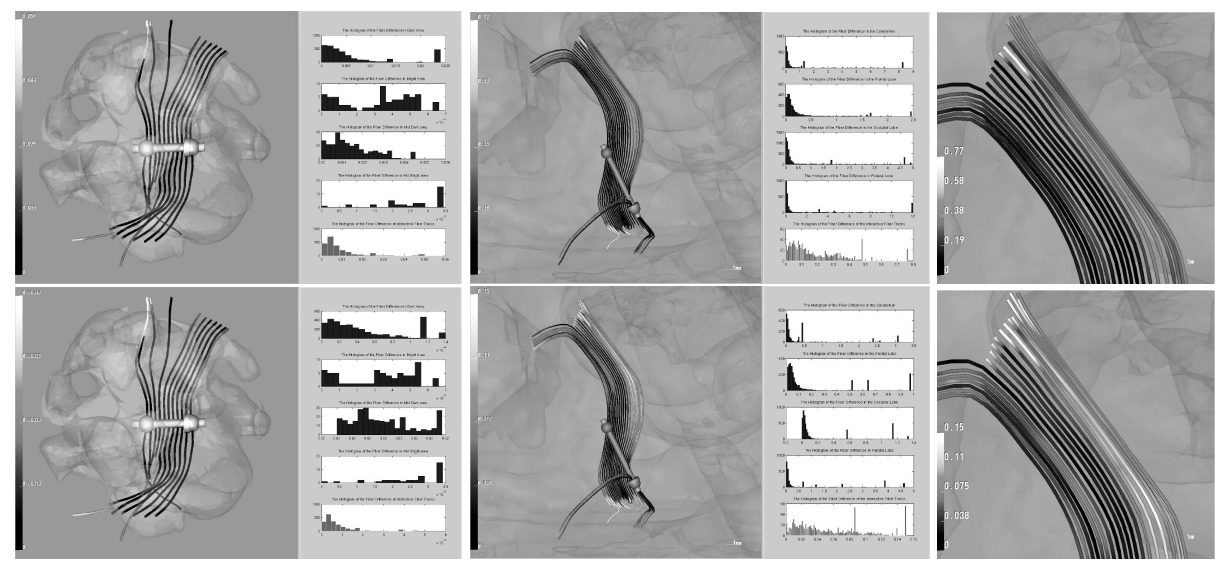

Fig. 5. The interactive visualization of local closest distance difference (top) and local area difference (bottom) of the synthetic data (left), monkey brain data (middle) and the zoom in view of the monkey brain data (right) 
quantification can be cast as a registration problem, therefore all of the other metrics already used in image registration, such as mutual information, may be useful for fiber bundle difference quantification. Furthermore, since the metrics we presented here are easily extended, we plan to compare q-ball and other higher order fiber tracking algorithms. We are also working with a group of neurologists to obtain anatomical axon tracks within the monkey brain as to compare histological ground truth of the brain connections with the tracking results of different algorithms. Finally, our interactive quantification and visualization toolkit may potentially be used as a tool for surgical planning aiding the further improvement of validation of Diffusion Tensor imaging.

\section{Acknowledgements}

The authors want to thank Suresh Venkatasubramanian, Yarden Livnat, J. Davison de St. Germain, Osama Abdullah and Tom Close for their useful discussions.

\section{References}

1. Basser, P., Mattiello, J., Lebihan, D.: Estimation of the effective self-diffusion tensor from the nmr spin echo. Journal of Magnetic Resonance, Series B 103 (1994) 247-254

2. Basser, P., Pajevic, S., Pierpaoli, C., Duda, J., Aldroubi, A.: In vivo fiber tractography using dt-mri data. Magnetic Resonance in Medicine 44 (2000) 625-632

3. Weinstein, D., Kindlmann, G., Lundberg, E.: Tensorlines: Advection-diffusion based propagation through diffusion tensor fields. (1999) 249-253

4. Lazar, M., Weinstein, D., Tsuruda, J., Hasan, K., Arfanakis, K., Meyerand, M., Badie, B., Rowley, H., Haughton, V., Field, A., Alexander, A.: White matter tractography using diffusion tensor deflection. Human Brain Mapping 18 (2003) 306-321

5. Parker, G., Wheeler-Kingshott, C., Barker, G.: Estimating distributed anatomical connectivity using fast marching methods and diffusion tensor imaging. IEEE Transactions on Medical Imaging 21 (2002) 505-512

6. Cheng, P., Magnotta, V., Wu, D., N.P., Moser, D., Paulsen, J., Jorge, R., Andreasen, N.: Evaluation of the gtract diffusion tensor tractography algorithm: A validation and reliability study. NeuroImage 31 (2006) 1075-1085

7. Friman, O., Farnebäck, G., Westin, C.F.: A bayesian approach for stochastic white matter tractography. IEEE Transactions on Medical Imaging 25 (2006) 965-978

8. Corouge, I., Fletcher, P.T., Joshi, S., Gouttard, S., Gerig, G.: Fiber tract-oriented statistics for quantitative diffusion tensor mri analysis. In: Proceedings Medical Image Computing and Computer Aided Intervention (MICCAI), Palm Springs, California (2005) 131-139

9. Goodlett, C.: Computation of statistics for populations of diffusion tensor images. Computation of Statistics for Populations of Diffusion Tensor Images (2009)

10. Wassermann, D., Bloy, L., Verma, R., Deriche, R.: A gaussian process based framework for white matter fiber tracts and bundles, applications to fiber clustering. In: Proceedings Medical Image Computing and Computer Aided Intervention (MICCAI Workshop), London (2009) 200-214

11. Johnson, C.: Top scientific visualization research problems. IEEE Transactions on Computer Graphics and Applications 24 (2004) 13-17

12. Brecheisen, R., Vilanova, A., Platel, B., Ter Haar Romeny, B.: Parameter sensitivity visualization for dti fiber tracking. IEEE Transactions on Visualization and Computer Graphics $\mathbf{1 5}$ (2009) 1441-1448 
13. Close, T., Tournier, J.D., Calamante, F., Johnston, L., Mareels, I., Connelly, A.: A software tool to generate simulated white matter structures for the assessment of fibre-tracking algorithms. NeuroImage 47 (2009) 1288-1300

14. Eiter, T., Mannila, H.: Computing discrete Frèchet distance. Technical Report CD-TR94/64, Christian Doppler Laboratory for Expert Systems, TU Vienna, Austria (1994)

15. Kantorovich, L.: On a problem of monge. Uspekhi Mat. Nauk. 3 (1948) 225-226

16. Vaillant, M., Glaunès, J.: Surface matching via currents. In: Proc. Information processing in medical imaging. Volume 19. (2005) 381-92

17. Durrleman, S.: Statistical models of currents for measuring the variability of anatomical curves, surfaces and their evolution. Thèse de sciences (phd thesis), Université de NiceSophia Antipolis (2010)

18. Joshi, S., Kommaraju, R.V., Phillips, J.M., Venkatasubramanian, S.: Matching shapes using the current distance. (Manuscript: arXiv:1001.0591) 